\title{
Severe Pubic Symphysis Diastasis Managed Conservatively: A Case Report and Review
}

\author{
Vijay Palvia1, Susan Kim², Holli Warholic ${ }^{1}$, James Anasti ${ }^{1}$ \\ ${ }^{1}$ Department of Obstetrics and Gynecology, St. Luke's University Health Network, Bethlehem, Pennsylvania, USA \\ ${ }^{2}$ Temple School of Medicine, Bethlehem, Pennsylvania, USA \\ Email: vijay.palvia@sluhn.org
}

How to cite this paper: Palvia, V., Kim, S. Warholic, H. and Anasti, J. (2017) Severe Pubic Symphysis Diastasis Managed Conservatively: A Case Report and Review. Case Reports in Clinical Medicine, 6, 120126.

https://doi.org/10.4236/crcm.2017.64010

Received: February 23, 2017

Accepted: April 27, 2017

Published: April 30, 2017

Copyright () 2017 by authors and Scientific Research Publishing Inc. This work is licensed under the Creative Commons Attribution International License (CC BY 4.0).

http://creativecommons.org/licenses/by/4.0/

\section{Open Access}

\begin{abstract}
Background: Pubic symphysis diastasis (PSD) is an uncommon complication of labor and delivery. Common risk factors of PSD include precipitous labor, rapid second stage of labor, intense uterine contractions, prior pelvic pathology, multiparity and macrosomia. Diagnosis is made clinically and confirmed by imaging. Management of PSD depends on the severity of symptoms and degree of symphysis separation. Standard therapy is conservative, but surgery may be needed in severe cases. Case Report: A 25-year-old female at term pregnancy presented in active labor and had a rapid second stage of labor without intravenous oxytocin or an epidural. She was subsequently diagnosed with severe PSD with a $5.5 \mathrm{~cm}$ separation. Her management included a pelvic binder, pain management, physical therapy, and serial imaging to monitor improvement. Discussion: In severe cases, surgery can be avoided in favor of conservative measures for the management of PSD. Multidisciplinary involvement with orthopedic surgery, radiology, physical therapy, and anesthesiology can play a vital role in optimal management. PSD may recur in future deliveries, but this does not preclude vaginal birth.
\end{abstract}

\section{Keywords}

Pubic Symphysis Diastasis

\section{Introduction}

The pubic symphysis is a midline joint which helps to stabilize the left and right halves of the pelvis while resisting tension, shearing, and compression forces. It is also the weakest point along the pelvic ring and subject to physiologic changes, particularly during pregnancy. Whereas symphyseal pain is a common occurrence, varying from $3 \%-20 \%$ of pregnancies [1], symphyseal rupture is uncommon with estimates ranging from 1:500 to $1: 30,000$ pregnancies [2]. This 
acute event most commonly occurs during the second stage of labor upon delivery of the newborn [3]. The implications of pubic symphysis diastasis (PSD) are serious and can result in long term complications if not immediately recognized.

PSD is a rare occurrence in obstetrics. A review of the current literature of the past 25 years using the PubMed database with key search terms ("pubic symphysis diastasis" and "vaginal delivery") returned only 12 case reports. In this report, we describe a patient who presented in spontaneous labor and later developed severe PSD. Informed consent was obtained from the patient to report this case. All necessary measures were taken to avoid reporting of any protected health information in accordance with the Health Insurance Portability and Accountability Act (HIPPA). Her labor course, clinical presentation, diagnosis, and management will be discussed here.

\section{Case Report}

A 25-year-old female, gravida 4 para 0212presented to her routine prenatal appointment complaining of contractions and leakage of fluid at 38 weeks and 3 days gestation. Upon further evaluation she reported leakage of clear fluid since 6:00 am, contractions every 2 minutes, and decreased fetal movement. On initial presentation, her vitals were stable, fetal heart tones were reactive with a baseline of 120 beats per minute, and contractions were every 3 to 5 minutes on to cometry. Cervical exam consisted of 5 to 6 centimeters $(\mathrm{cm})$ dilation, $70 \%$ effacement, -1 station of fetal head. Ferning and nitrazine tests were negative.

Her pregnancy was significant for group beta streptococcus (GBS) colonization of the genital tract, two prior preterm vaginal deliveries, anemia, and $\mathrm{O}$ negative blood type. Her past medical history is uncomplicated but her surgical history is significant for omphalocele repair (1990), exploratory laparotomy for small bowel obstruction (1998), cholecystectomy (2003), and appendectomy (1990).

Upon admission to the labor and delivery unit, routine labs were ordered and intravenous penicillin was started for indication of GBS infection. Her hemoglobin on admission was $9.9 \mathrm{~g} / \mathrm{dL}$ (reference range 11.5 to $15.4 \mathrm{~g} / \mathrm{dL}$ ). She was then monitored for expectant management of her labor. At 10:00 pm, her cervical exam indicated she was only $6 \mathrm{~cm}$ dilated. She subsequently underwent amniotomy with clear fluid during this exam. She was reexamined at 12:30 am and was found to be significantly uncomfortable during her contractions. Her cervical exam showed minimal change at only 6 to $7 \mathrm{~cm}$ dilation. She declined an epidural for pain management and oxytocin for augmentation. Over the next 2 hours, she continued to demonstrate significant discomfort during her contractions.

At approximately 2:00 am, she reached complete cervical dilation with obvious fetal descent and had a strong urge to push. During her second stage of labor, there was difficulty with patient positioning and compliance with coordinated pushing. Further descent of the fetal head occurred rapidly over the next few minutes. Upon crowning of the fetal head, an audible sound was appreciated 
likely originating from the patient's right hip. There was obvious concern for hip injury. The baby delivered spontaneously without difficulty weighing 6 pounds and 14 ounces. The placenta was delivered without difficulty and bleeding was well controlled. Estimated blood loss at delivery was $300 \mathrm{~mL}$.

Shortly after delivery, a neurovascular exam of the patient's lower extremities showed inability of bilateral hip flexion, pain on palpation of hip joints, and severe vulvar edema. She had appropriate dorsiflexion and plantar flexion of feet, intact sensation, and 2+ dorsalis pedis pulse. A Foley catheter was inserted for concern of urinary retention in light of recent trauma and vulvar edema. An anterior-posterior hip $\mathrm{x}$-ray showed a $5.5 \mathrm{~cm}$ diastasis of the pubic symphysis along with mild widening of the sacroiliac joints (Figure 1). No other degenerative changes, dislocations, or fractures were noted on $\mathrm{x}$-ray, however, prominent soft tissue swelling of the vulva and vagina was present. Orthopedic surgery recommended pelvic binder placement with serial tightening, serial hip X-rays, physical therapy, enoxaparin for deep venous thromboembolism prophylaxis, and pain management. The T-POD Pelvic Stabilization Device (Pyng Medical) was employed as the pelvic binder. The possibility of surgery was considered if conservative measures failed.

On postpartum day 1, pain was well controlled and serial tightening of the pelvic binder was tolerated by the patient. Repeat hip x-rays showed gradual reduction of the pubic symphysis diastasis from $5.5 \mathrm{~cm}$ to $1.6 \mathrm{~cm}$ by postpartum day 1 (Figure 2). She had marked improvement in mobilization and ambulation with physical therapy by post partum day 2 . By postpartum day 3 , her pain, mobility, and vulvar edema further improved. She was discharged on postpartum day 3 with the pelvic binder and was to follow up as outpatient with her obstetrician and orthopedic surgeon.

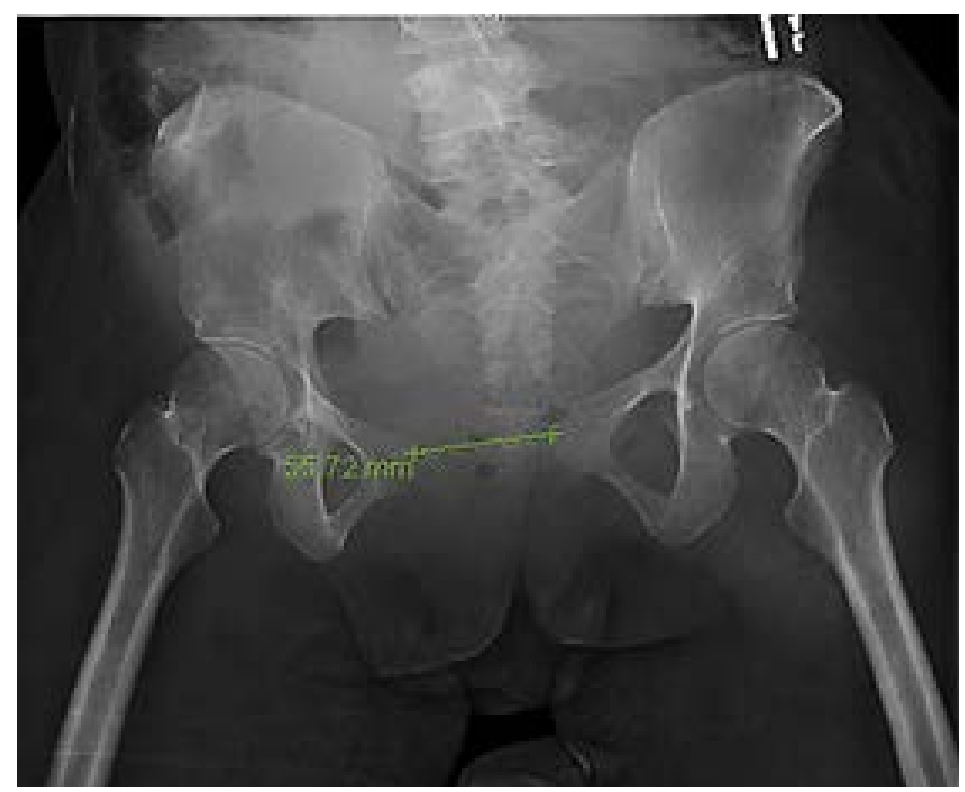

Figure 1. Marked pubic symphysis diastasis of $5.5 \mathrm{~cm}$ with mild widening of the sacroiliac joints. 


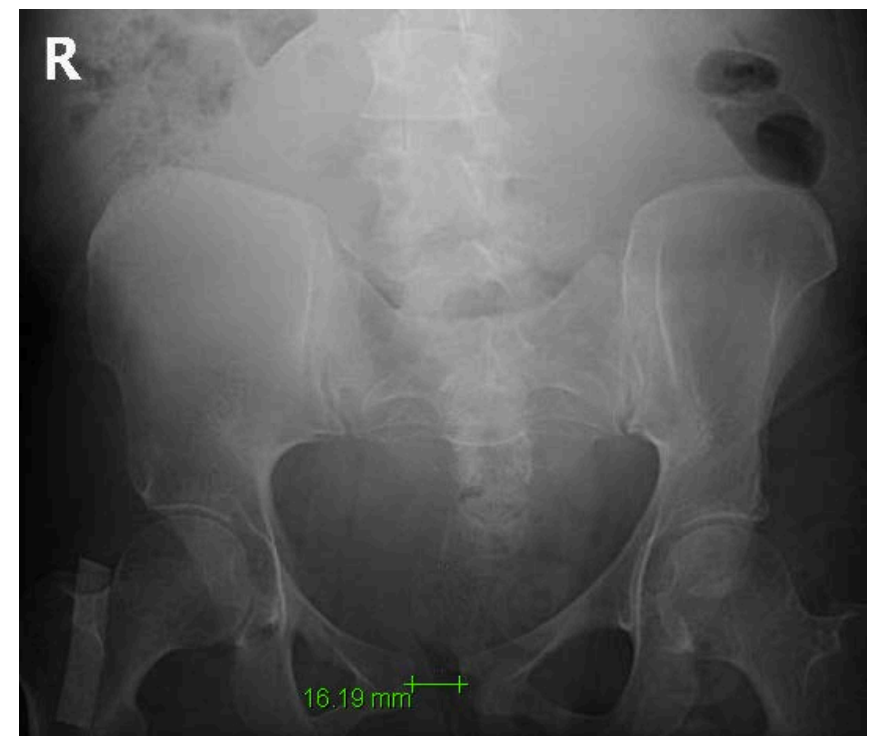

Figure 2. Dramatic improvement in diastasis with serial tightening of pelvic binder.

\section{Discussion}

Our patient is a rare but classic presentation of severe pubic symphysis diastasis. Her prenatal and antepartum care provided no clear indication of being considered an at-risk patient for this rare obstetrical complication. Her risk factors for PSD, discussed below, became evident after her diagnosis. Subsequent management required consultation with Orthopedic Surgery to determine best route of care. Fortunately, conservative management proved to be an adequate approach for her recovery. Proper care of this patient requires a detailed knowledge of the anatomy, biophysiology, clinical presentation, diagnostic testing, and therapeutic approaches for pubic symphysis diastasis.

Anatomically, the pubic symphysis is a vital component of the bony pelvis anatomy. It is a non-synovial fibrocartilaginous joint, which connects the superior rami of the bilateral pubic bones. The pubic symphysis consists of an interpubic disc with an articular surface that connects to both pubic bones. Further support is provided by four variable ligaments: the anterior, inferior, superior, and posterior ligaments. Collectively, the ligaments support the pubic symphysis and allow for its multidirectional movement. Vascularity to the pubic symphysis is supplied by branches of the obturator artery, inferior epigastric arteries, internal pudendal arteries, and medial circumflex femoral artery. Innervation comes from the branches of the pudendal, genitofemoral, iliohypogastric, and ilioinguinal nerves [1]. Fortunately, there was no evidence of neurovascular trauma in our patient.

The width of the pubic symphyseal joint is between $2.6 \mathrm{~mm}$ and $4 \mathrm{~mm}$. It can widen by 3 to $4 \mathrm{~mm}$ during the course of pregnancy. A healthy joint is highly resistant to separation and requires significant exacerbation in order to rupture. Multiparous women undergo weakening of their ligamentous support, which further worsens during the course of pregnancy. Ligamentous laxity is mediated 
by relaxin and progesterone, which cause collagen degradation and remodeling by inducing expression of matrix metalloproteinases. The risk of rupture further increases during childbirth due to variable stress on these ligaments. In our case, it is evident that these ligaments underwent significant stress during the conclusion of the second stage of labor [1].

Multiple risk factors for symphyseal rupture have been identified. The most common factors being fetal macrosomia and cephalopelvic disproportion. Other associated factors include rapid descent of the fetal head, precipitous labor, short second stage of labor, forceful abduction of the thighs, or difficult forceps delivery [4]. Degenerative joint disease due to prior pelvic trauma, arthritis, osteomalacia, chondromalacia, and congenital dysplasia has also been associated with rupture [5]. Although the majority of patients are multiparous, this is not a consistent finding as primigravida separations have occurred [6]. In regard to our patient, she was multiparous and her labor course was notable for rapid descent of the fetal head, short second stage of labor, and uncoordinated positioning and pushing.

Finally, epidural anesthesia can be a risk factor, but the correlation is less clear. An epidural can increase time of labor, which contradicts with the risk factor of precipitous labor. Theoretically, the anesthesia may cause numbness to the point where the patient will forcefully abduct her thighs further or bear down stronger during contractions to increase joint load. In contrast, lack of regional anesthesia can result in severe pain and discomfort during the second stage of labor, which can result in uncoordinated positioning and pushing [5].

Clinical manifestations of pubic symphysis diastasis are often immediately apparent with acute onset of symptoms. Patients commonly experience a sharp, tearing pain localized to the pubic symphysis with or without an audible click or snap. Patients will often be unable to walk or have abnormal gait. Although less common, there may be associated injuries such as hemorrhage, hemodynamic instability, sacroiliac dislocation, sacral fracture, lumbosacral plexopathy, bladder injury, and death [7]. A pathognomonic feature of symphyseal rupture is the presence of severe pubic pain with compression of both greater trochanters towards the midline. The patient may also have an inability to flex the hip joint. Less commonly, the patient may have a palpable gap between the pelvic bones [8].

PSD is generally a clinical diagnosis. In instances where a practitioner may be uncertain whether the patient is experiencing pubic symphyseal dysfunction versus actual rupture, the diagnosis may be supplemented by either radiograph or ultrasound [5]. Diastasis is considered severe if symphyseal separation is greater than $2.5 \mathrm{~cm}$ on imaging. Notably, the width of separation does not correlate with severity of symptoms [9]. If there is high suspicion for injury to vasculature, soft tissues, adjacent organs, or additional bony structures, then a computed tomography (CT) scan should be performed [7]. A pelvic x-ray was ordered for our patient to confirm PSD, however, a CT scan was not needed based on her clinical presentation.

Routine management of pubic symphysis diastasis is largely conservative. 
However, select patients may be considered for surgical management: patients with severe diastasis ( $>25 \mathrm{~mm}$ ), failed conservative management, or pelvic instability (hemorrhage, open rupture, soft tissue damage). Despite a separation of $5.5 \mathrm{~cm}$, a conservative approach was pursued after consulting with the orthopedic surgical team. If surgery was needed, the patient would have been a candidate for open reduction and internal fixation. In most cases, surgery is rarely required [10].

Conservative management includes pain analgesia, bed rest, pelvic support with a binder, ambulation with a walker or crutches, and a gradual physical therapy regimen. A pelvic binder can be placed around the hips and tightened gradually to allow for reduction of the pubic symphysis separation. Nonsteroidal anti-inflammatory agents and opiates are the most common medications for pain analgesia. Physical therapy is a major component of timely recovery. A graded exercise regimen and proper evaluation by a physical therapist will help obtain pain-free ambulation. Walkers and crutches maybe helpful in these circumstances for assisted ambulation [5]. Involving the orthopedic surgeon and physical therapist was critical in our patient's quick recovery.

Most patients have resolution of their symptoms by 6 months but some may have persistent chronic pain for 12 months or beyond. Repeating a hip x-ray is appropriate 6 - 10 weeks after the injury to assess proper healing and approximation of the pubic bone. For symptomatic patients, continued physical therapy will benefit the patient for pain relief, muscle strengthening, and quicker return to activities of daily living. Patients should be encouraged to reduce non-essential weight bearing and to rest when possible.

Appropriate follow up with an obstetrician regarding pain management and counseling for future pregnancy is recommended. Prior history of pelvic fracture does not preclude trial of labor or vaginal delivery. Nonetheless, these patients have a higher incidence of subsequent cesarean delivery based on history [7].

\section{Conclusion}

This case presents multiple learning points regarding management of severe pubic symphysis diastasis. Understanding the major risk factors and clinical manifestations of PSD can help ascertain a diagnosis. Precautions such as pain management, coordinated positioning and pushing, careful forceps delivery, and identifying cephalopelvic disproportion can mitigate these risks. This case involved a conservative approach to management of severe pubic symphysis diastasis and demonstrates the importance of prompt assessment of the patient's clinical condition to optimize her recovery. Another learning point includes the value of a multidisciplinary approach to the patient's management. We believe our case will benefit other clinical teams by placing confidence in non-surgical management, especially in scenarios of severe PSD.

\section{References}

[1] Becker, I., Woodley, S.J. and Stringer, M.D. (2010) The Adult Human Pubic Sym- 
physis: A Systematic Review. Journal of Anatomy, 217, 475-487.

https://doi.org/10.1111/j.1469-7580.2010.01300.x

[2] Schwartz, Z., Katz, Z. and Lancet, M. (1985) Management of Puerperal Separation of the Symphysis Pubis. International Journal of Gynaecology and Obstetrics, 23, 125-128. https://doi.org/10.1016/0020-7292(85)90056-6

[3] Rustamova, S., Predanic, M., Sumersille, M. and Cohen, W.R. (2009) Changes in Symphysis Pubis Width during Labor. Journal of Perinatal Medicine, 37, 370-373. https://doi.org/10.1515/JPM.2009.051

[4] Shnaekel, K.L., Magann. E.F. and Ahmadi, S. (2015) Pubic Symphysis Rupture and Separation during Pregnancy. Obstetrical \& Gynecological Surgery, 70, 713-718. https://doi.org/10.1097/OGX.0000000000000247

[5] Jain, N., Sternberg, L.B. (2005) Symphyseal Separation. Obstetrics and Gynecology, 201, 1229-1232. https://doi.org/10.1097/01.AOG.0000149744.82912.ea

[6] Valsky, D.V., Anteby, E.Y., Hiller, N., Amsalem, H., Yagel, S. and Hochner-Celnikier, D. (2006) Postpartum Pubic Separation Associated with Prolonged Urinary Retention Following Spontaneous Delivery. Acta Obstetricia et Gynecologica Scandinavica, 85, 1267-1269. https://doi.org/10.1080/00016340600608501

[7] Amorosa, L.F., Amorosa, J.H., Wellman, D.S., Lorich, D.G. and Helfet, D.L. (2013) Management of Pelvic Injuries in Pregnancy. Orthopedic Clinics of North America, 44, 301-315. https://doi.org/10.1016/j.ocl.2013.03.005

[8] Leadbetter, R.E., Mawer, D. and Lindow, S.W. (2006) The Development of a Scoring System for Symphysis Pubis Dysfunction. Journal of Obstetrics and Gynecology, 26, 20-23. https://doi.org/10.1080/01443610500363915

[9] Owens, K., Pearson, A. and Mason, G. (2002) Symphysis Pubis Dysfunction-A Cause of Significant Obstetric Morbidity. European Journal of Obstetrics and Gynecology and Reproductive Biology, 105, 143-146.

https://doi.org/10.1016/S0301-2115(02)00192-6

[10] Osterhoff, G., Ossendorf, C., Ossendorf-Kimmichi, N., Zimmermann, R., Wanner, G.A., Simmen, H.P., et al. (2012) Surgical Stabilization of Postpartum Symphyseal Instability: Two Cases and a Review of the Literature. Gynecologic and Obstetric Investigation, 73, 1-7. https://doi.org/10.1159/000331055

\section{Scientific Research Publishing}

Submit or recommend next manuscript to SCIRP and we will provide best service for you:

Accepting pre-submission inquiries through Email, Facebook, LinkedIn, Twitter, etc. A wide selection of journals (inclusive of 9 subjects, more than 200 journals)

Providing 24-hour high-quality service

User-friendly online submission system

Fair and swift peer-review system

Efficient typesetting and proofreading procedure

Display of the result of downloads and visits, as well as the number of cited articles

Maximum dissemination of your research work

Submit your manuscript at: http://papersubmission.scirp.org/

Or contact crcm@scirp.org 